\title{
TOWARDS OPTIMAL SPECTRAL AND SPATIAL DOCUMENTATION OF CULTURAL HERITAGE. COSCH - AN INTERDISCIPLINARY ACTION IN THE COST FRAMEWORK
}

\author{
F. Boochs ${ }^{\text {a }}$, *, A. Bentkowska-Kafel ${ }^{\text {b }}$, C. Degringy ${ }^{c}$, M. Hautta-Kasari $^{d}$, S. Rizvic ${ }^{e}$, R. Sitnik ${ }^{f}$, A. Tremeau ${ }^{g}$ \\ a i3mainz, University of Applied Sciences Mainz, Lucy Hillebrand Str. 2, 55128, Mainz, Germany - \\ boochs@geoinform.fh-mainz.de \\ ${ }^{\mathrm{b}}$ Department of Digital Humanities, King's College London, 26-29 Drury Lane, London WC2B 5RL, United Kingdom - \\ anna.bentkowska@kcl.ac.uk \\ c Haute Ecole de Conservation-restauration, Espace de l'Europe 11, Neuchâtel, 2000, Neuchâtel, Switzerland - \\ christian.degrigny@he-arc.ch \\ ${ }^{\mathrm{d}}$ School of Computing, University of Eastern Finland, P.O. Box 111, 80101 Joensuu, Finland - \\ markku.hautakasari@uef.fi \\ ${ }^{\text {e }}$ Faculty of Electrical Engineering, University of Sarajevo, Zmaja od Bosne bb, Sarajevo 71000, Bosnia-Herzegovina - \\ srizvic@etf.unsa.ba \\ ${ }^{\mathrm{f}}$ Faculty of Mechatronics, Warsaw University of Technology, 02-525 Warsaw, Poland - \\ r.sitnik@mchtr.pw.edu.pl \\ ${ }^{g}$ Laboratoire Hubert Curien UMR 5516, Université Jean Monnet - Saint Etienne, 18 rue B. Lauras, Saint-Etienne, 42000 France - \\ alain.tremeau@univ-st-etienne.fr
}

KEY WORDS: multispectral data, colour, 3D data, multi-sensor data, registration, visualisation, analysis, cultural heritage applications, collaborative research

\begin{abstract}
:
This paper introduces the aims and early activities of Colour and Space in Cultural Heritage (COSCH), an interdisciplinary European network of experts in the latest optical measuring techniques and electronic imaging applied to documentation of artefacts. $\mathrm{COSCH}$ is a forum open to organisations, institutions and companies interested in collaboration within the emerging field of precise spectral and spatial imaging techniques, in physical and chemical sciences applied to cultural heritage objects, as well as in research and applications to conservation and art-historical analysis of such objects. COSCH started in November 2012. Funded by COST, an intergovernmental framework for European Cooperation in Science and Technology, COSCH networking activities enable knowledge exchange and coordination of nationally-funded research on a European level with occasional contribution of experts from other countries. Funding has been made available for four years (2012-2016). Participation is open to researchers across a wide range of disciplines, including computer scientists and museum professionals, art historians and academics in heritage-related fields. COSCH is a trans-domain Action (TD1201) of the COST Domain Materials, Physics and Nanosciences (MPNS) which facilitates and promotes innovation in material science. The work of $\mathrm{COSCH}$ is defined in the Memorandum of Understanding between the COST Office and the Chairman of COSCH. The Memorandum is available from http://www.cost.eu/domains_actions/mpns/Actions/TD1201 alongside the latest progress report and other documents. The scientific work draws on earlier and current research of the participants and is organised around the following areas: spectral and spatial object documentation; algorithms and procedures; analysis and restoration of surfaces and objects of material culture; visualisation of cultural heritage objects and its dissemination. Up-to-date information about $\mathrm{COSCH}$ activities, including its scientific and training programmes, abstracts of presentations and a list of participants, can all be found on the Action website at http://www.cosch.info.
\end{abstract}

\section{INTRODUCTION}

\subsection{Background}

True, precise and complete documentation of artefacts is essential for conservation and preservation of cultural heritage $(\mathrm{CH})$. By ensuring access to the best possible documentation of artefacts, Colour and Space in Cultural Heritage (COSCH) aims to contribute to the enhanced understanding of material cultural heritage and thus support its long-term preservation. Documentation of cultural heritage involves collaboration of researchers, scientists and professionals from multiple disciplines and industries. An urgent need has been identified to promote interdisciplinary research, development and application of non-contact optical measurement techniques (spectral and spatial) - adapted to the needs of material heritage documentation - on a concerted European level, in order to record, analyse, understand, protect, preserve, model, virtually reproduce and publish important cultural heritage in Europe and beyond.

Research in this field has typically relied on nationally-funded projects with insufficient interaction between experts and stakeholders. COSCH aims to provide a stimulating framework for articulating and clarifying problems, sharing solutions and skills, standardising methodologies and protocols, encouraging 
a common understanding, widening applications and dissemination of expert knowledge across relevant disciplines. The Action aims to foster open standards for state-of-the-art documentation of material cultural heritage. By stimulating research into simplified usage of high-resolution optical techniques $\mathrm{COSCH}$ aims to define good practices and widen their adoption within cultural heritage sectors and education.

\subsection{Interdisciplinary Collaboration}

In this context a close collaboration between cultural sectors and scientists is paramount to ensure reciprocal understanding of the requirements of documentation and preservation of material culture and establish how technology should develop to meet the needs. This approach requires bridging "two cultures" - science and the arts - and overcoming many existing barriers in order to gain a better understanding of discrete methodologies, concepts and research practices. COSCH aims to communicate scientific and technical research in a way that may benefit non-scientists. Through the development of case studies and other collaborative initiatives, arts and humanities specialists participating in the $\mathrm{COSCH}$ Action will have the opportunity to better understand the state-of-the-art techniques for precision spectral and spatial documentation in order to explore the huge potential of non-contact methods in their fields. Scientists specialising in optical technologies will be able to learn through dialogue about the requirements for documentation and analysis of historic architecture, archaeological sites and valuable heritage objects, such as paintings, sculptures and coins, in order to optimise current techniques and ensure that future developments go in the right direction and meet expectations. Material scientists, physicists and chemists, alongside conservators, will be able to provide the theoretical and scientific expertise in connecting optical data with material properties of artefacts.

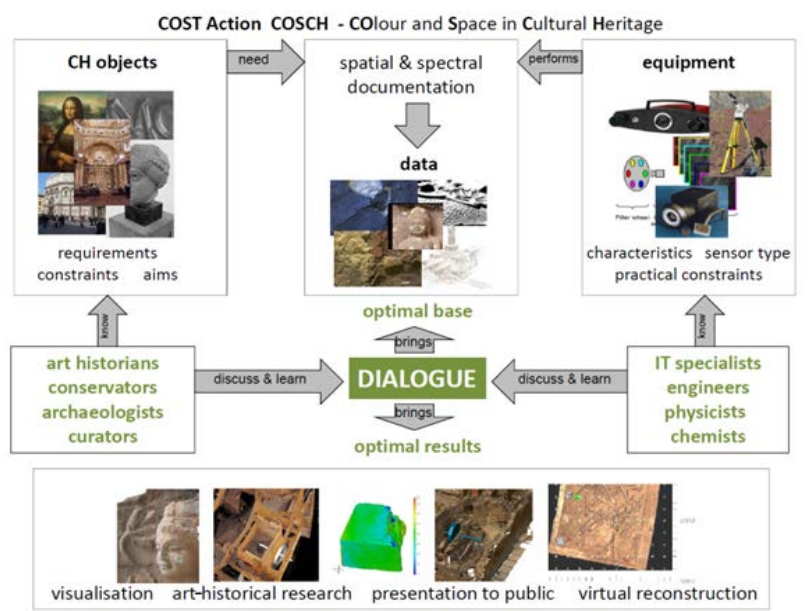

Figure 1. An overview of the COSCH Action

It should be noted that $\mathrm{COSCH}$ is a trans-domain Action (TD1201) of the COST Domain Materials, Physics and Nanosciences (MPNS) which facilitates and promotes innovation in material science. The involvement in $\mathrm{COSCH}$ of material scientists is of particular importance owing to a strong connection between the surface material of heritage objects, their visual appearance and techniques suitable for optical documentation. The involvement of chemists is equally helpful owing to the dependence of spectral reflectance on the chemical composition of an object; this is of great interest when investigating ageing processes.
Through this coordinated Action scientific progress is expected in particular in the area of spatio-spectral acquisition, processing, rendering and quality evaluation for heritage documentation. Arts and Humanities researchers, museum professionals and educators will benefit from examples of accurate records of artefacts, once access to these is made available to them, while scientists developing optical recording techniques will benefit from the guidance, standards and principles widely used in the cultural sector, such as the UNESCO and ICOMOS charters on documentation and preservation of heritage (both material and digital). The London Charter for 3D visualisation of cultural heritage promotes the principles of historical and scientific reliability and intellectual transparency of virtual artefacts. It also deals with the notoriously difficult question of ambiguity when analysing incomplete historical evidence (material and intangible) and recommends a number of measures for ensuring that visualisation-based documentation of cultural heritage is accurate and reliable.

All this requires effective communication and exchange of information based on openness to novel solutions which, when borrowed from a field which is not one's own, may sometimes be difficult. Mutual understanding may be achieved through exposure to and discussion of interdisciplinary research questions. Integrating discrete scientific and academic disciplines represented within $\mathrm{COSCH}$ poses a challenge to all participants. An art historian certainly does not feel at ease when contributing to research within the COST domain of Materials, Physics and Nanosciences. Equally, it may not be easy for a specialist in optical measurements to consider hypothetical or ambiguous solutions that often need to be addressed, and recorded, in virtual representations of historical artefacts. Progress can be expected when interdisciplinary research relies on open-minded international collaboration and maximally integrates the richness and variety of knowledge and expertise available and communicated globally.

\section{COSCH ACTIVITIES}

COSCH activities rely on the work of five expert groups, a training and mobility programmes (internships, schools and exchange visits called Short Term Scientific Missions), and a wide range of external dissemination activities.

\subsection{Working Groups}

The core work of COSCH is organised in five thematic working groups (WG):

WG 1: Spectral object documentation

WG 2: Spatial object documentation

WG 3: Algorithms and procedures

WG 4: Analysis and restoration of $\mathrm{CH}$ surfaces and objects WG 5: Visualisation of $\mathrm{CH}$ objects and its dissemination

The Spectral Object Documentation Group focuses on two main tasks: (1) identification, characterisation and testing of spectral imaging techniques in the visible and near infra-red field and (2) identification, characterisation and testing of imaging techniques beyond the visible and short wave radiation. The Spatial Object Documentation Group looks in particular at (1) identification of the main 3D scanning techniques suitable for use in cultural heritage objects, and (2) analysis and comparison of the different $3 \mathrm{D}$ scanning techniques. The main 
tasks of the Algorithms and Procedures Group are (1) registration processes (acquisition, filtering and view integration) and (2) integration of multi-sensor data. The forth Working Group is concerned with the analysis and restoration of cultural heritage surfaces and objects. Its tasks include (1) identification, structuring and implementation of typical use cases, and (2) development of guidelines. The fifth group works on visualisation of cultural heritage objects and its dissemination, looking in particular at (1) identification, planning, implementation and testing of typical applications of visualisation within cultural heritage domains, and (2) a further development of visualisation techniques.

$\mathrm{COSCH}$ has identified many interesting questions. Spectral imaging techniques undergo a comparable change in technology as do spatial imaging techniques. New developments in optical techniques lead to new means of splitting light resulting in new characteristics for instruments dedicated to monitor the optical spectrum for imaged surfaces. It is therefore necessary to explore the limits and advantages of the actual instruments in this waveband.

The physical and chemical composition of surfaces is an important factor determining the interaction with light and reflection behaviour. As not all of these factors have an impact in the visible spectrum, instruments exist which are able to measure beyond this radiation. It is necessary to exploit and qualify also these types of instruments because they are often essential for the analysis of the surface composition.

The most common 3D scanning techniques used in digitisation of artefacts are structured light, stereoscopic imaging and laser scanning, although also a number of other metrology techniques, such as time-of-flight and interferometry, provide useful information in certain environmental conditions. An evaluation of different techniques applied to 3D scanning is essential to show conditions of its applicability and explain the limitations.

The actual performance of the techniques available for different types of cultural heritage objects must be analysed in great depth, based on the identified setups and available expertise. The goal is to analyse the advantages and limitations of these techniques depending on the deciding characteristics, and to address the identified drawbacks by stimulating new developments.

Optical systems provide data for one field of view. As objects in general have to be monitored using many views, techniques are needed to merge individual views. Traditional techniques have physical impact on the surface, which for most cultural heritage objects is unacceptable. It is therefore necessary to identify, evaluate and classify available techniques for fusion of views.

Optical techniques for applications for cultural heritage objects are contactless and carry certain information content, based on the interaction between light and surface. This interaction depends on the wavelength and the sensor used. Thus, information content can be extended by using different instruments with varying spectral characteristics. A number of integrating steps are required, which have to be categorised and classified.

Measured data provide valuable content which should be stored and made available to anyone using this information in cultural heritage applications. However, due to characteristics introduced by the vendors of instruments or software there exist many barriers preventing from easy access. This situation has to be addressed and remedied, especially in interdisciplinary and multi-sensoral working fields.

Surface analyses based on various optical, physical or chemical characteristics may serve diverse purposes. Surface characteristics are subject to the interaction with the optical radiation and the process of data capture. It is therefore crucial for $\mathrm{COSH}$ to identify critical factors affecting these processes and to establish a reliable knowledge base. Similarly, it is important to identify and define the impact of the instrumentation on the quality of results. Critical steps in the analytical process should be identified depending on the purpose of the undertaken analysis.

Cultural heritage objects can be visualised in many different ways and for different purposes. Applications range from edutainment and relatively simple presentation to the general public to accurate 3D records surveyed for specialist, professional research and conservation. Each purpose bears on the quality expected from visualisation. Characteristics of the data have to be identified and the visualisation processes structured and implemented accordingly, ensuring access and longevity of the final output.

Development of innovative and reliable visualisation techniques, including integration of multi-sensor data as base for enhanced exploitation of information pertaining to shape and colour, as well as other object data and metadata is critical.

\subsection{COSCH Meetings}

$\mathrm{COSCH}$ meets twice a year. A two or three day event consists of academic sessions organised by the Working Groups, a plenary discussion, a meeting of the $\mathrm{COSCH}$ Management Committee, demonstrations of technology and events open to a wider non-COSCH audience.

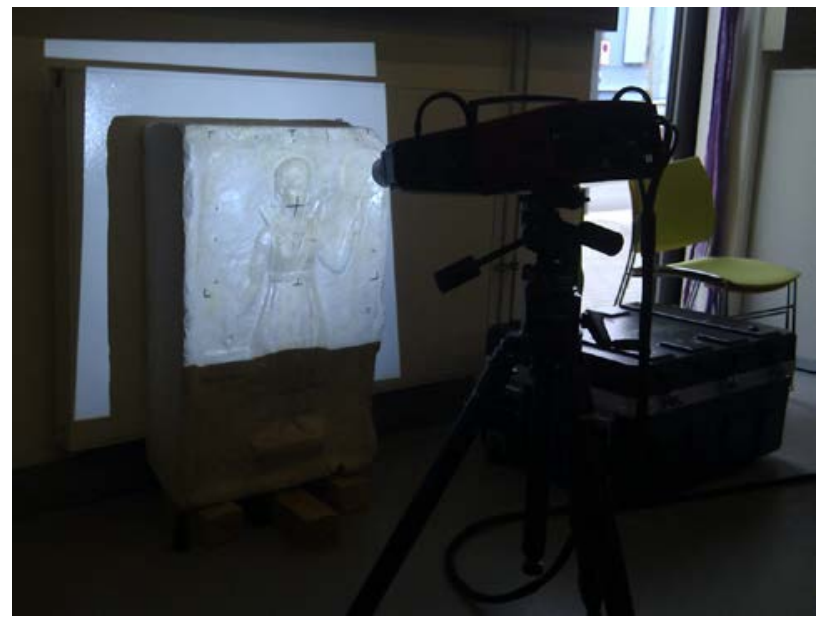

Figure 2. Demonstration of 3D laser scanning at the Institute for Spatial Information and Surveying Technology, i3Mainz

The first COSCH meeting was held at the FH-Mainz University of Applied Sciences, Mainz, Germany, on 26th and 27th March 2013. The programme was intensive and consisted of 24 presentations by international speakers, demonstrations of 3D technologies and doctoral research projects currently being undertaken at the host institution, as well as a public defense of 
a PhD thesis by Camille Simon Chane on integration of spatial data and spectral data acquisition in the context of information generation applied to heritage conservation. $\mathrm{COSCH}$ is not funded for carrying out new scientific research but rather to draw upon projects made possible through funding from other sources. COSCH offers a forum for constructive discussion and a number of instruments for facilitating and stimulating the exchange and wider adoption of specialist knowledge.

The second COSCH meeting will be held on 23-25 September 2013 in London, UK. It will be hosted by the Department of Digital Humanities at King's College London. Associated events will include an imaging workshop organised by the University College London (UCL) experts in Geomatics Photogrammetry, 3D Imaging and Metrology. They will demonstrate four areas in imaging techniques for capture and rendering of cultural heritage object: Arius 3D colour laser scanner; low-cost and hand-held 3D scanning techniques; a dome for Polynomial Texture Mapping/Reflectance Transformation Imaging, as well as applications of these imaging techniques in the form of interactive exhibits at the UCL Petrie Museum of Egyptian Archaeology, which combine high resolution 3D colour scanning of museum objects with rich investigative storytelling.

The programmes of both meetings and selected abstracts of presentations are available on the $\mathrm{COSCH}$ website.

\subsection{COSCH Internships}

$\mathrm{COSCH}$ is proactive in engaging researchers across Europe in the scientific objectives of the Action. COSCH fosters collaboration. Short-Term Scientific Missions (STSM) serve this aim. They enable scientists to stay in an institution or laboratory in another country (eligible to receive COST funding) for a period of up to three months to carry out theoretical or practical investigations of material cultural objects by collecting and analysing spatial and/or spectral data; to learn a new technique or to take measurements using instruments and/or methods not available in their own institution/laboratory. This scheme has already proven to be popular, particularly among young scientists. COSCH was successful in increasing the number of STMSs originally agreed for 2013. Nine applications have been approved. The reports on completed missions will be made available on the COSCH website.

\subsection{Future Activities}

The development of networking, information exchange, dissemination and capacity building measures, within the framework established by COST for trans-domain actions, will continue throughout the duration of the $\mathrm{COSCH}$ Action. The second meeting of COSCH in London will see the launch of the Think Tank for Early Stage Researcher on 24 September 2013; their first academic session is planned for the following day. The Technical University in Warsaw is organising the first COSCH Training School which will take place in October 2013. A number of scientific publications are planned based on the rich programme of academic and technical activities. The content of the COSCH website will continue to be enhanced in the course of the Action.

\section{CONCLUSIONS}

\subsection{COSCH Objectives}

Main objectives of the work coordinated by the COSCH Action are:

1. Theoretical identification and practical exploration of important characteristics of instruments and their potential impact on data quality, usability and information content with respect to typical surfaces of cultural heritage objects.

2. Identification and definition of typical application/object requirements and their impact on the characteristics of data to be able to support these applications.

3. Theoretical analysis and practical investigation of typical and necessary processing tasks and their potential or real impact on quality and information content of results.

4. Selection of typical applications and/or objects to be subject of implementation of optimal processing chains, from data capture up to the final results, guided by all the interdisciplinary expertise available to $\mathrm{COSCH}$.

5. Establishment of the conceptual and practical frameworks for multisensory data acquisition, its implementation and evaluation.

6. Development of recommendations for solution providers as well as end users. These recommendations are expected to facilitate a deeper integration of optical technology into applications to cultural heritage objects through an improved correlation between optical means and requirements.

$\mathrm{COSCH}$ is continuously open to new members and invites participation of specialists with relevant research interests and organisations willing to collaborate in this interesting and important interdisciplinary area. Anyone interested in joining $\mathrm{COSCH}$ should contact the Management Committee by following the instruction for new participants available on the Action website (http://www.cosch.info).

\subsection{Selected Bibliography}

Bentkowska-Kafel, A. 2007. Needs of the 3D Visualisation Community. A report produced for the 3D Visualisation in the Arts Network commissioned by the UK Joint Information Systems Committee (JISC), London, http://3dvisa.cch.kcl.ac.uk/reports.html (8 Jul. 2013)

Bentkowska-Kafel, A., Denard. H. (eds) 2012. Paradata and Transparency in Virtual Heritage, Farnham: Ashgate.

Brandmüller, J., Moser, H. 1962. Einführung in die Ramanspektroskopie. Steinkopff, Darmstadt, Wissenschaftliche Forschungsberichte. Naturwissenschaftliche Reihe 70.

Bunsch, E., Sitnik, R., Michoński, K. 2011. Art Documentation Quality in Function of 3D Scanning Resolution and Precision, Computer Vision And Image Analysis Of Art Ii. Proceedings of The Spie, Vol. 7869, Edited by Stork, D. G.; Coddington, J.; Bentkowska-Kafel, A. Article Id. 78690d, 11 pp. 
COSCH | Colour and Space in Cultural Heritage website http://www.cosch.info (8 Jul. 2013)

COST (European Cooperation in Science and Technology) webpage about the COSCH Action (incl. key documents) http://www.cost.eu/domains_actions/mpns/Actions/TD1201 (8 Jul. 2013)

Cucci, C., Picollo, M., Vervat, M. 2012. Trans-illumination and trans-irradiation with digital cameras: potentials and limits of two imaging techniques used for the diagnostic investigation of paintings, Journal of Cultural Heritage, 13(1), pp. 83-88.

Demetriou, M.-L., Hardeberg, J. H., Adelmann, G. 2011. Computer-Aided Reclamation of Lost Art - A New Concept, Proceedings of Gjøvik Color Imaging Symposium, Gjøvik, Norway, pp. 551-560.

Grøn, O., Palmer, S., Stylegar, F.-A., Esbensen, K., Kucheryavski, S., Aase, S. 2011. Interpretation of archaeological small-scale features in spectral images, Journal of Archaeological Science, 38(9), pp. 2024-2030.

Lasarte, M. de, Arjona, M., Vilaseca, M., Pujol, J. 2010. Influence of the number of samples in a training set on the accuracy of color measurement and spectral reconstruction, Journal of Imaging Science and Technology 54(3), pp. 305011-30501-10(10).

London Charter for the Computer-based Visualisation of Cultural Heritage http://www.londoncharter.org (8 Jul. 2013)

Mansouri, A., Śliwa, T., Hardeberg, J. H., Voisin, Y. 2008. Reflectance estimation using PCA and wavelet basis decomposition, Color Research and Application, 33(6), pp. 485-493.

Simon, C., Schütze, R., Boochs, F., Marzani, F. 2012. Asserting the Precise Position of 3D and Multispectral Acquisition Systems for Multisensor Registration Applied to Cultural Heritage Analysis. In: Advances in Multimedia Modeling, Proceedings of the 18th International Conference on MultiMedia Modeling, Klagenfurt, Austria, 4-6 January 2012. Edited by K. Schoeffmann et al., Springer-Verlag Berlin Heiderberg, pp. 597-608.

\subsection{Acknowledgements}

The Authors acknowledge the support of the European Cooperation in Science and Technology and wish to thank all contributors to the work of COSCH (COST Action TD1201).

8 July 2013 